\title{
Entreprises manufacturières ; TIC et chaine logistique L'impact des TIC sur l'avantage concurrentiel de la chaine logistique dans les entreprises manufacturières Algériennes
}

\section{Résumé:}

Aujourd'hui, on accorde une importance de plus en plus accrue au rôle joué par les technologies de l'information et de la communication dans l'optimisation de la gestion de la chaine logistique. En nous basant sur les travaux précurseurs menés sur le rôle des TIC dans la création de l'avantage concurrentiel de Porter et Millar (1985) $)^{(1)}$, nous avons mené une étude empirique sur un échantillon de 227 petites et moyennes entreprises (PME) activant dans le secteur de la manufacture. Les résultats de l'enquête ont permis de voir que les PME ayant introduit des outils informatiques dans leur logistique, ont vu leur croissance s'accroitre et leur position concurrentielle s'améliorer considérablement.

Mots clés : TIC, chaine logistique, création de valeur pour le client, avantage concurrentiel

$$
\begin{aligned}
& \text { لقد أصبح: البوم لتكنولوجيا المعلومات والاتصال دور كبير } \\
& \text { في تحسين إدارة سلسلة نوريد المؤسسات. استتاداً إلى ل } \\
& \text { الاعمال الأولية حول دور تكنولوجيا المعلومات و الاتصال } \\
& \text { في خلق الميزة التنافسية لـ (1985) Porter et millar, } \\
& \text { أجرينا دراسة تجريبية لعينة من } 227 \text { مؤسسة صغيرة } \\
& \text { ومتوسطة الحجم في قطاع التصنيع وأظهرت النتائج } \\
& \text { المتحصل عليها أن الثركات التي أدخلت أدوات تكنولوجيا } \\
& \text { المعلومات في شماتها اللوجستية، شهدت زيادة } \\
& \text { نمو ها وتحسين موقعها التنافسي إلى حد كبير. }
\end{aligned}
$$

\author{
AMARA Nesrine \\ Ecole Nationale Superieure de \\ Management- pôle universitaire de \\ Koléa. TIPAZA
}

\begin{abstract}
Introduction :
À partir des années 90, de nombreuses mutations sont survenues dans le monde de la gestion. On a assisté à une forte mondialisation des échanges, un développement rapide des technologies, un raccourcissement du cycle de vie du produit et des clients de plus en plus exigeants. Dans un tel contexte, les entreprises se sont vues obligées de chercher des stratégies qui leur permettent d'apporter une valeur supérieure, aux yeux des clients.
\end{abstract}


En effet, alors qu'il est devenu difficile de construire et de maintenir un avantage concurrentiel à travers une stratégie basée sur les prix, d'autres stratégies de différenciation sont développées au sein des entreprises pour attirer la clientèle : valorisation des compétences, développement de l'image de marque, développement de produits innovants et flexibilité organisationnelle notamment, à travers une gestion de la chaine logistique performante.

Considérée comme activité contemporaine, la fonction logistique s'est intensifiée, en passant d'une logique fonctionnelle concentrée sur la gestion opérationnelle des flux physiques à une logique systémique basée sur une gestion stratégique des flux physiques et informationnels. Le crédit de développement d'une telle approche dite "'gestion de la Chaine logistique" est à attribuer, dans une large mesure, à Porter (1985)(2) et à Porter et Millar (1985), qui alertent les dirigeants et les professionnels sur l'importance centrale de coordonner et de synchroniser entre les différents maillons de la chaine logistique, afin d'augmenter la valeur créée pour le client en termes de qualité, de coûts et de délais.

La concertation entre les maillons de la chaine logistique, a été rendue possible grâce à diversité de méthodologies et d'outils, proposés par des entreprises leaders dans le domaine. Les technologies de l'information et de la communication représentent les outils incontournables, qui ont permis aux entreprises d'entrer dans l'ère de la numérisation et de l'automatisation, afin de s'adapter rapidement aux nouvelles demandes des clients et aux opportunités du marché. Les recherches dominantes entreprises dans le domaine, de type "étude de cas", développent les méthodologies de mise en place d'outils informatiques de grandes entreprises mondiales (Ripoll, 1997 ${ }^{(3)}$; Delfmann et Gehring, $2003^{(4)}$; Morvan, 2005 $\left.5^{(5)} \ldots\right)$. Ces solutions informatiques ont réduit sensiblement les cycles du processus qui part de la recherche pour aboutir à la livraison du produit en passant par les étapes intermédiaires de conception, de planification, d'approvisionnement, de production, et de vente. Elles ont ainsi permis de fournir la bonne information, à la bonne personne, au bon moment et au meilleur coût afin de prendre la bonne décision qui augmentera la productivité de l'entreprise.

Pour le cas de l'Algérie, la prise de conscience des entreprises, de la nécessaire maitrise de la gestion de la chaine logistique a tardé à se dessiner du fait du monopole exercé par les grandes entreprises nationales au détriment des entreprises privées. En termes de recherches académiques, on constate une prédominance d'études de type "monographie", qui s'intéressent à la gestion de la chaine logistique des grandes entreprises agro-alimentaires (Boudahri, $2013^{(6)}$; Berraki, 2014 ${ }^{(7)}$ ). 
Nous avons mené une enquête de terrain sur 227 PME manufacturières pour, d'une part, étudier l'importance accordée par les entreprises algériennes à l'introduction des TIC dans leur chaine logistique, et d'autre part, analyser le rôle des TIC «technologies de l'information et de la communication» dans l'optimisation stratégique de la chaine logistique des entreprises algériennes. Il s'agira de voir si les entreprises qui utilisent l'outil informatique dans la gestion de leur logistique interne et externe, réalisent une meilleure performance/compétitive.

Pour répondre à cette question, nous avons tout d'abord tenté de décrire les recherches qui ont mis l'accent sur l'importance des TIC dans la création de valeur pour le client et par conséquent, l'obtention d'un avantage concurrentiel. Ensuite, nous avons présenté les résultats de l'enquête qui se distinguent des recherches antérieures entreprises dans le domaine par son caractère empirique qui s'appuie sur un échantillon composé de PME et non pas sur une étude monographique.

Les résultats de l'enquête permettent d'appréhender non seulement le rôle des TIC dans la croissance des grandes entreprises et des PME mais aussi dans l'amélioration de leur position concurrentielle.

I. Les fondements théoriques de l'impact de l'introduction des TIC dans la chaine logistique sur la création de valeur pour le client :

Pour analyser l'impact de l'introduction des TIC dans la chaine logistique sur la compétitivité des entreprises, il semble nécessaire de donner d'abord un aperçu sur la logistique. Ensuite, il s'agira de présenter les principales recherches axées sur l'importance stratégique de la chaine logistique dans la compétitivité des entreprises et enfin de mettre en évidence les recherches qui considèrent les TIC comme source de cette compétitivité.

\section{Aperçu sur la chaine logistique.}

D'origine militaire, la logistique vient du mot grec «LOGISTIKOS», qui signifie l'art de raisonnement et de calcul. Elle est apparue dans les entreprises au cours des années 60. A l'origine, elle répondait à un problème de coûts de distribution qui affectent lourdement la structure du prix de revient du produit acheté par le client final. Basée sur la gestion des flux physiques (marchandises), la logistique fut structurée à cette époque, selon une approche fragmentée où chaque fonction (approvisionnement, production et distribution) tente de trouver des solutions logistiques techniques indépendamment des autres fonctions.

Une telle approche fonctionnelle a conduit à l'apparition de problèmes de coordination, traduits dans les faits par une performance intrinsèque d'une fonction au détriment d'une autre. Pour corriger ces problèmes, la logistique est passée d'une logique fragmentée à une logique transversale où des fonctions coordonnées concourent à une meilleure circulation des flux physiques, en vue de réaliser une meilleure performance du couple coûts- service client. 
Au début des années 80 , une nouvelle vision intégratrice et systémique dite "chaîne logistique" a vu le jour. Utilisé pour la première fois par Oliver et Weber $(1982)^{(8)}$, ce terme a été défini par de nombreux chercheurs comme étant un ensemble d'installations ou d'entités (achat, production et distribution) échangeant des flux (physique, informationnel, financier). La chaine logistique est constituée d'une part d'un processus amont qui couvre la planification de la production, les approvisionnements, la fabrication et la gestion des stocks et d'autre part d'un processus aval qui couvre la planification aval, la gestion des réseaux de distribution, le transport et la livraison des produits finis (Beamon, 1998 $)^{(9)}$. La logistique n'est pas alors perçue comme une démarche opérationnelle mais comme une démarche qui tend à court et moyen terme à piloter et gérer les flux physiques à travers les flux informationnels associés (chaine logistique).

Ce n'est qu'à partir des années 90, que la vision stratégique de la logistique s'est répandue dans le monde de l'entreprise. On ne parle plus de "logistique" mais de " gestion de la chaine logistique. L'avantage concurrentiel est ainsi construit à travers une intégration des unités organisationnelles tout au long d'une chaine logistique qui part des fournisseurs pour aboutir aux clients finaux (interne et externe à l'entreprise) dans un ensemble coordonné des flux physiques, informationnels et financiers.

\section{Chaine logistique et compétitivité.}

C'est au début des années 60 , avec le développement de la fonction marketing, qu'on a commencé à avoir une vision de création de valeur pour le client. Chaque fonction tentée d'une manière fragmentée, de faire de la logistique en gérant les flux physiques pour réaliser les niveaux de service promis aux clients à un coût acceptable. L'intérêt pour la création de valeur pour le client s'est accru par la suite à travers l'émergence d'une vision intégratrice dite chaine logistique (achat, production et distribution) où on pilote les flux physiques à travers des flux informationnels.

La 'valeur' est un terme qui a connu plusieurs tentatives de définitions. Dans le cadre de cette étude, nous retenons la définition proposée par Lorino (1997) qui stipule que "La valeur est le jugement porté par la société (notamment le marché et les clients potentiels) sur l'utilité des prestations offertes par l'entreprise comme réponse à des besoins " ${ }^{(10)}$. Cette définition montre que la valeur créée peut avoir une multitude de destinataires mais c'est l'offre qui satisfait les besoins du client, qui est mise en avant. Une telle vision s'inscrit dans une approche globale de la logistique (Fabbe-Costes, 2002) ${ }^{(11)}$, où la création de valeur pour le client représente un garant de création de valeur pour l'ensemble des autres acteurs de la chaine logistique, notamment en termes d'avantage concurrentiel. 
Le modèle de l'avantage concurrentiel développé par Porter $(1986)^{(12)}$ a permis à la logique de création de valeur pour le client, de prendre une nouvelle tournure. On ne parle plus d'intégration des trois fonctions achat, production et distribution mais d'intégration de l'ensemble des fonctions au sein de l'entreprise, qui concourent à la création de valeur pour le client, source d'avantage concurrentiel pour l'entreprise. Il a ainsi mis en avant, le concept de 'chaine de valeur' qui permet de décomposer la firme en activités pertinentes au plan de la stratégie (activités principales et activités de soutien) dans le but de comprendre le comportement des coûts et les potentielles sources existantes de différenciation.

Au début des années 90, "la gestion de la chaine logistique" a vu le jour comme fonction stratégique cherchant à gérer les relations entre les maillons de la chaine logistique, pour une meilleure satisfaction du client. La prise en compte de la nécessité d'adopter cette approche intégratrice, s'est imposée au milieu des années 90 et au début des années 2000 suite à l'intérêt porté par les chercheurs et les praticiens, à une telle thématique. Ils ont présenté la gestion de la chaine logistique comme source d'optimisation de la chaine de valeur en terme de réduction des coûts, (Giunipero et Brand, 1996 ${ }^{(13)}$; Harrington, 1999 ${ }^{(14)}$; Palevich, $\left.1997^{(15)}\right)$, d'amélioration du service à la clientèle, d' amélioration de la marge bénéficiaire (Alber et Walker, $1997^{(16)}$ ), de prix , de variété de la gamme, de rapidité de la livraison, de service au client,(Laurentie et al. 2000) ${ }^{(17)}$, de flexibilité (Fabbe- Costes, Meschi $2000^{(18)}$ ), de fiabilité des processus mis en place, de rapidité dans les prestations (Jobin et Friel, $2001^{(19)}$ ), de développement de projets innovants (Primo et Amundson, 2002(20)), de qualité de service et de performance en délai et en réactivité (Nakhla, 2006 ${ }^{(21)}$ ).

Pour le cas de l'Algérie, rare sont les recherches initiées dans le contexte de la chaine logistique. Comme il a été relevé plus haut, les seules recherches entreprises concernent des études monographiques sur l'optimisation de la performance d'entreprises activant dans le secteur agro-alimentaire (Boudahri, $2013^{(22)}$; Berraki, 2014 ${ }^{(23)}$ ).

\section{TIC, chaine logistique et compétitivité.}

De nombreuses recherches ont mis l'accent sur le rôle d'une chaine logistique optimisée sur la création de valeur. Une optimisation qui s'opère selon Fernandes $(2007)^{(24)}$, grâce à la prise en compte préalable des exigences du client et à l'apport d'une gestion efficiente de la logistique. Il s'agira d'assurer au mieux la coordination et la synchronisation des flux physiques et informationnels de la chaine, qui impliquent des acteurs aux interdépendances diverses (Blanquart, Carbone et Zeroual, 2011) $)^{(25)}$. 
Depuis le début des années 1980, la recherche accrue de compétitivité a conduit les entreprises à réviser, à réinventer et à automatiser l'ensemble de leurs processus de décision, de conception, de production et de distribution. C'est, pour ces entreprises, une nécessité absolue, d'autant plus que la gestion des flux informationnels est devenue une question primordiale pour une meilleure optimisation de l'action collective amont et aval d'une chaine logistique. Divers outils et solutions technologiques (logiciels, télécommunications, informatique embarquée) ont été ainsi introduits dans les entreprises pour le pilotage informationnel des marchandises tout au long de la chaîne logistique. Les technologies dites les outils Info logistiques, ont joué un rôle moteur dans le développement des innovations logistiques dédiées principalement à la gestion et aux échanges d'informations, au sein de la chaîne logistique : code-barres, logiciels de planification et d'exécution, systèmes EDI (Échanges de Données Informatisées), et technologies d'identification et de traçabilité, magasin automatisé, fabrication assistée par ordinateur, fichier clientèle informatisé.

Diverses approches managériales d'évaluation de l'impact de l'intégration des TIC dans l'organisation, peuvent être recensées: approche par l'analyse concurrentielle, approche par l'alignement stratégique, approche orientée processus...

Pour notre part et dans le cadre de cette recherche, nous nous basons sur l'approche par l'analyse concurrentielle qui met l'accent sur le rôle des technologies de l'information et de la communication dans la modification du jeu concurrentiel. On peut citer comme auteurs de cette approche: Parsons $(1983)^{(26)}$ pour qui les TIC permettent de modifier les rapports de forces de l'entreprise avec ses concurrents et Ives et Leamonth (1984) ${ }^{(27)}$ qui a écrit sur le rôle des TIC dans l'optimisation de la gestion des relations avec les clients et fournisseurs. On ne peut parler de rôle des TIC sans faire appel aux travaux précurseurs dans ce domaine, de Porter et Millar (1985) ${ }^{(28)}$. Ils présentent les TIC comme source d'avantage concurrentiel non seulement en termes de coûts mais aussi de différenciation.

Pour construire leur modèle, sur lequel se base d'ailleurs la présente recherche, Porter et Millar (1985) ont mis en avant le rôle des TIC dans la chaine de valeur.

Figure 1: La chaine de valeur

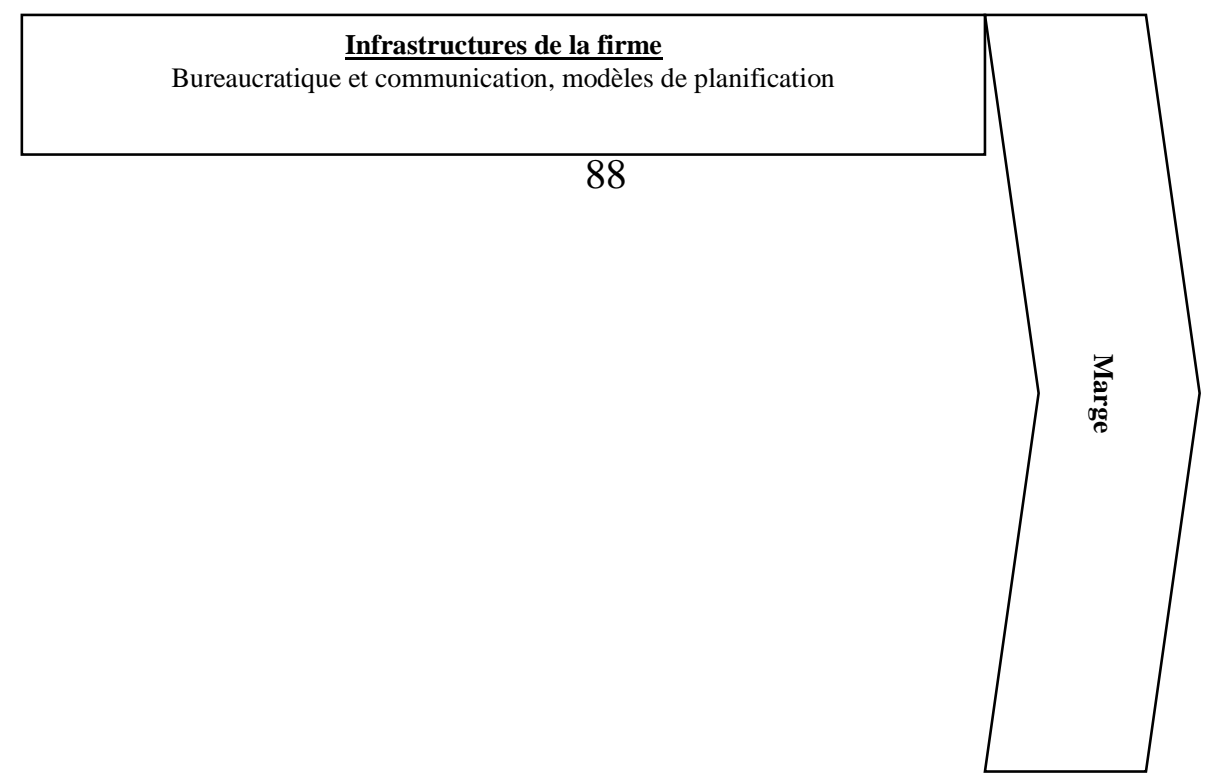


Entreprises manufacturières ; TIC et chaine logistique L'impact des TIC sur l'avantage concurrentiel de la chaine logistique dans les entreprises manufacturières Algériennes

\begin{tabular}{|c|c|c|c|c|}
\hline \multicolumn{5}{|c|}{$\begin{array}{l}\text { Gestion des ressources humaines } \\
\text { Système d'affectation, base de données des compétences }\end{array}$} \\
\hline \multicolumn{5}{|c|}{$\frac{\text { Développement technologique }}{\text { Conception assistée par ordinateur }}$} \\
\hline \multicolumn{5}{|c|}{ Échange de données informatisées (EDI) } \\
\hline$\frac{\text { Logistique }}{\text { interne }}$ & & $\frac{\text { Logistique }}{\text { externe }}$ & Commercia & $\underline{\text { Services }}$ \\
\hline $\begin{array}{c}\text { Magasin } \\
\text { automatiqu } \\
\mathrm{e}\end{array}$ & $\begin{array}{l}\text { Fabrication } \\
\text { assistée par } \\
\text { ordinateur }\end{array}$ & $\begin{array}{l}\text { Prise de } \\
\text { commande } \\
\text { en temps } \\
\text { réel }\end{array}$ & $\begin{array}{l}\text { Vente par } \\
\text { terminaux } \\
\text { (portables } \\
\text { essentiellem } \\
\quad \text { ent) }\end{array}$ & $\begin{array}{l}\text { Maintenanc } \\
\text { e à distance }\end{array}$ \\
\hline
\end{tabular}

Source: Porter M.E. et Millar V. (1985), "How information gives you competitive advantage", Harvard Business Review, volume 63, n4, avril 1985.

Chaque activité est constituée d'une composante physique (tâches pour l'exécution de l'activité) et d'une composante informationnelle (la collecte, la transformation et la diffusion des informations nécessaires pour exécuter l'activité). L'interdépendance entre les activités, conduit à la nécessité d'une coordination entre elles. Selon les auteurs, ceci a été rendu possible grâce aux TIC qui ont la capacité de s'infiltrer dans la chaine de valeur des entreprises et de coordonner les différentes activités de base et de soutien.

L'importance des TIC, comme outils d'amélioration de la performance de la chaine logistique, a été largement discutée et mise en évidence par de nombreuses études développées par des praticiens qui ont tenté de présenter les meilleures pratiques d'optimisation de la chaine logistique de grandes entreprises (Ripoll, 1997 ; ARCIS, 1999 ; Delfmann et Gehring, 2003 ; Morvan, 2005 ...).

Ainsi, Ripoll (1997) ${ }^{(29)}$ a présenté le cas de Procter \& Gamble, l'initiateur de l'ECR (Réponse Efficace au Consommateur) aux États Unis pour démontrer l'impact de l'intégration des TIC dans l'optimisation de la chaine logistique amont. Les TIC ont permis à Procter \& Gamble d'augmenter considérablement leur chiffre d'Affaires, par la résolution des problèmes de disponibilité des produits, d'optimisation des rayonnages. 
Arcis (1999) ${ }^{(30)}$ a démontré l'importance de l'acquisition de la technologie ERP (Enterprise Resource Planning), pour l'adaptation rapide aux nouvelles demandes des clients et aux opportunités du marché. Elle a permis la bonne gestion des approvisionnements, de la production, des ventes et de la distribution des produits et services.

Delfmann et Gehring (2003) ${ }^{(31)}$ ont publié une étude internationale réalisée par McKinsey \& Company et le Département planification opérationnelle et logistique de l'université de Cologne, auprès d'une cinquante de prestataires de services et d'entreprises industrielles et de distribution. Ils ont pu démontrer que l'utilisation de l'outil informatique dans le management de la chaine logistique est beaucoup plus important dans le cas des entreprises performantes : soit prés de $70 \%$ de ces entreprises utilisent un système de commande automatisé et $96 \%$ d'entre elles possèdent un système de planification de la production (PPM). L'étude a conclu que la performance de la gestion de la chaine logistique aval de ces entreprises, se traduit non seulement par une flexibilité dans la gestion des stocks mais également par un raccourcissement du temps de traitement des commandes des clients de $20 \%$.

Ainsi, Morvan $(2005)^{(32)}$ a tenté d'analyser les avantages apportés par l'implémentation d'un ERP visant à optimiser la performance de la chaine logistique amont et aval chez HILTI, un important fournisseur dans le secteur du BTP. Il présente les ERP comme source d'une meilleure circulation des flux dans la chaine logistique et un meilleur service au client.

En ce qui concerne les recherches algériennes entreprises sur le rôle des TIC dans l'optimisation de la gestion de la chaine logistique, à notre connaissance, il ne semble pas exister de telles études. La seule recherche que nous avons pu recenser, s'intéresse au rôle du logiciel Arena dans l'évaluation de la performance de la chaine logistique.

La disponibilité d'un fonds documentaire qui traite de l'impact de l'introduction des hautes technologies (ECR, ERP...) sur l'optimisation de la chaine logistique et l'absence de recherches algériennes dans le domaine, plaident pour la nécessité d'entamer une recherche qui s'inscrirait dans une perspective de l'approche de Porter et Millar (1985) et qui prendrait en considération quelques dimensions prises en compte par les praticiens (croissance des entreprises et position concurrentielle). Il s'agira de voir le rôle joué par l'informatisation des différentes phases de la chaine logistique aussi bien amont qu'aval, sur la création de valeur pour le client, source d'avantage concurrentiel pour l'entreprise.

\section{Organisation de la chaine logistique : \\ 1. Méthodologie :}


Aujourd'hui, on accorde une importance de plus en plus accrue, au rôle joué par les TIC dans l'optimisation de la gestion de la chaine logistique, en vue d'obtenir un avantage concurrentiel. Un tel constat nous a motivé à adopter une approche empirique par l'examen de deux principaux aspects :

- L'importance accordée par les entreprises algériennes à l'introduction des TIC dans leur chaine logistique ;

- L'impact induit par l'introduction des TIC au sein de la chaine logistique, sur la compétitivité des entreprises algériennes.

La recherche que nous nous proposons de mener, se démarque des autres études entreprises en Algérie dans le domaine, par son caractère empirique. Elle se fonde sur un échantillon d'entreprises algériennes.

Pour mener notre enquête, nous avons pris un échantillon de départ de 1000 entreprises. Nous avons obtenu 414 réponses; soit un taux de réponses de $41,4 \%$. Nous avons d'abord sélectionné les 324 entreprises qui activent dans le secteur manufacturier, ensuite nous avons pris 256 entreprises qui disposent des TIC ; soit : 227 PME et 29 grandes entreprises. Pour la présente recherche, nous nous sommes intéressés aux 227 PME.

Trois principaux volets ont été retenus pour réaliser notre enquête :

- Organisation de la chaine logistique. En premier lieu, il s'agira de présenter la configuration de la chaine logistique au sein de ces entreprises. Ensuite, il faudra analyser le degré de pénétration des technologies de l'information et de la communication, dans la chaine logistique. A ce niveau, il s'agira de voir quels sont les domaines de la logistique privilégiés par les entreprises pour l'intégration des TIC. Cette analyse permettra de calculer ' 'l'Indice de pénétration des TIC dans la chaine logistique par nombre de domaine".

- Contribution de l'informatisation de la chaine logistique dans la croissance des entreprises, où il s'agira d'étudier l'impact produit par l'introduction des TIC dans la chaine logistique, sur la croissance des entreprises, exprimée en termes de chiffre d'affaires et de valeur ajoutée ;

- Informatisation de la chaine logistique et position concurrentielle des entreprises, où il sera analysé le rôle tenu par l'introduction des TIC dans la chaine logistique, sur la position concurrentielle.

Pour analyser les données nous avons utilisé 'SPSS" et 'Excel'.

\section{La chaine logistique : configuration}

a. Structure des fournisseurs: 


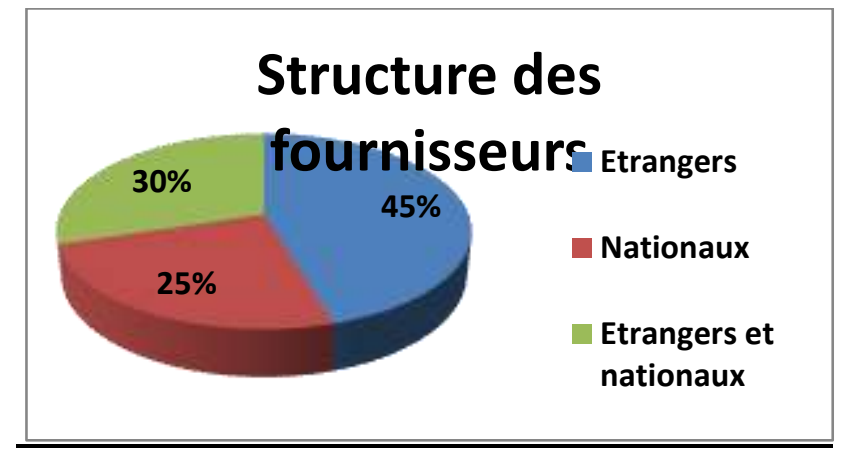

En ce qui concerne l'approvisionnement en matières premières, on peut constater que les PME de notre échantillon, dépendent pour $45 \%$ du marché extérieur. L'approvisionnement local intervient pour seulement $25 \%$.

\section{b. Structure de la clientèle :}

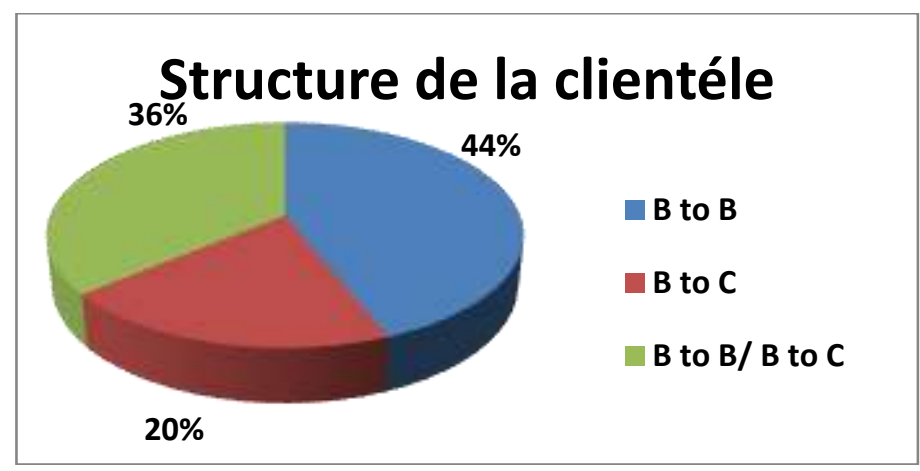

Une analyse de la structure de la clientèle, permet de voir qu'il existe une diversité de clientèles. En effet, on constate que $44 \%$ des PME activent dans le secteur B to B, c'est-à-dire qu'elles ont une clientèle industrielle; $20 \%$ s'adressent directement aux consommateurs finaux et $36 \%$ qui activent aussi bien dans le B to B que dans le B to $\mathrm{C}$.

\section{c. Transport :}


Entreprises manufacturières ; TIC et chaine logistique L'impact des TIC sur l'avantage concurrentiel de la chaine logistique dans les entreprises manufacturières Algériennes

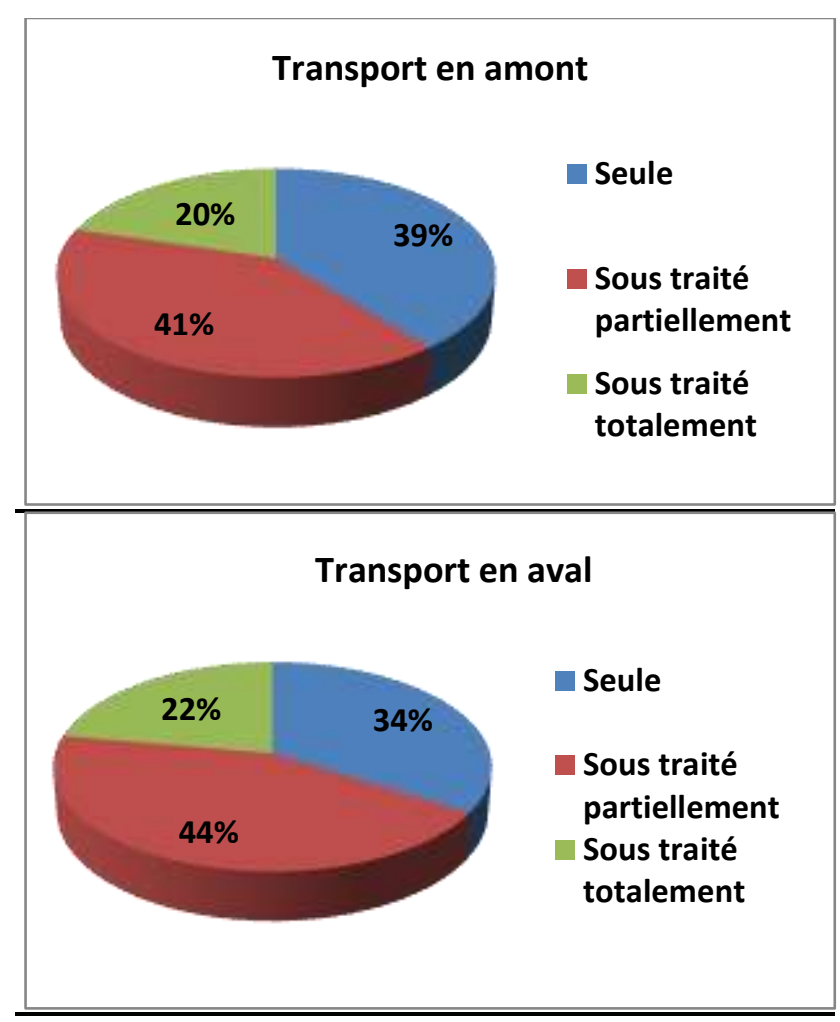

La majorité des PME de notre échantillon sous-traitent l'activité de transport aussi bien en amont qu'en aval ; soit : $61 \%$ des entreprises recourent à la soustraitance pour le transport de la matière première et $66 \%$ de l'échantillon soustraitent le transport des produits finis vers le consommateur final. Ceci peut être perçu comme une stratégie adoptée par les PME pour diminuer les charges de transport qui serait générées par la gestion et la maintenance d'une flotte de transport.

\section{d. Canaux de vente :}




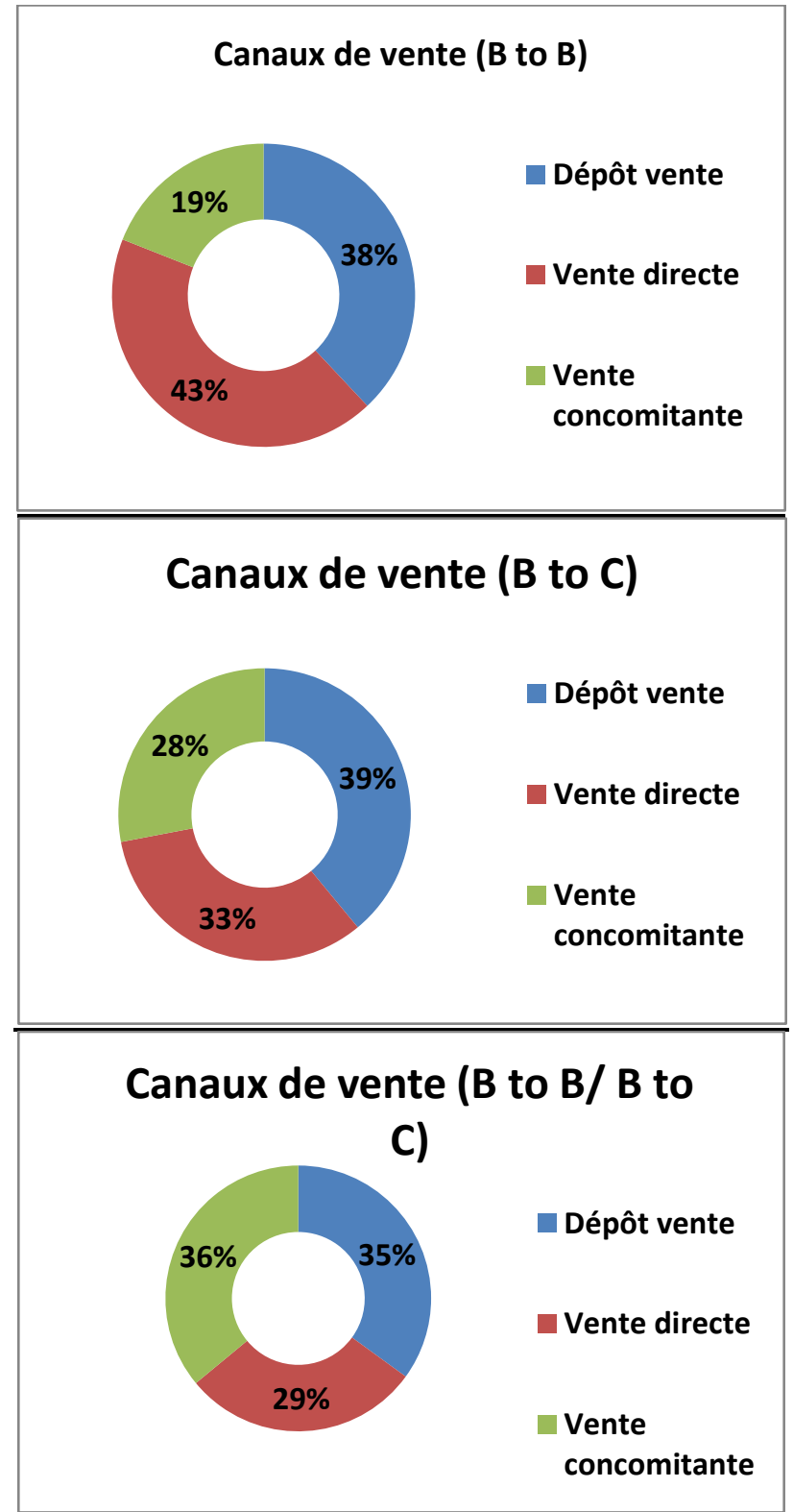

Pour les canaux de vente, on constate que les PME qui activent dans B to B font beaucoup plus appel à la vente directe sur le marché (43\%). Celles qui activent dans le $\mathrm{B}$ to $\mathrm{C}$ font appel à des intermédiaires (39\%) et une grande part des PME qui activent dans le $\mathrm{B}$ to $\mathrm{b}$ ou dans le $\mathrm{b}$ to $\mathrm{C}$ font appel aussi bien au dépôt vente $(35 \%)$ qu'à la vente concomitante.

e. L'importance de la logistique: 
Entreprises manufacturières ; TIC et chaine logistique L'impact des TIC sur l'avantage concurrentiel de la chaine logistique dans les entreprises manufacturières Algériennes

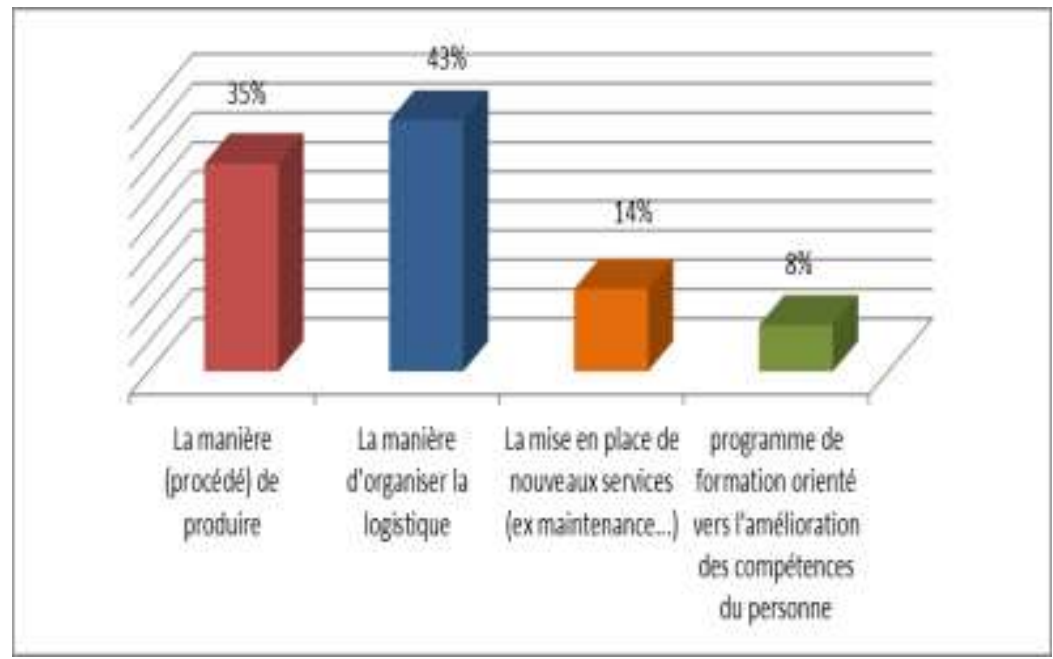

En ce qui concerne, les actions initiées par les entreprises, ces trois dernières années, on constate que l'organisation de la logistique est une préoccupation des PME algériennes (34\% des entreprises). Ensuite, viennent la manière de produire, la mise en place de nouveaux services et l'organisation de programmes de formation, orientés vers l'amélioration des compétences du personnel.

3. Pénétration des TIC dans la chaine logistique :

a. La facture électronique : 

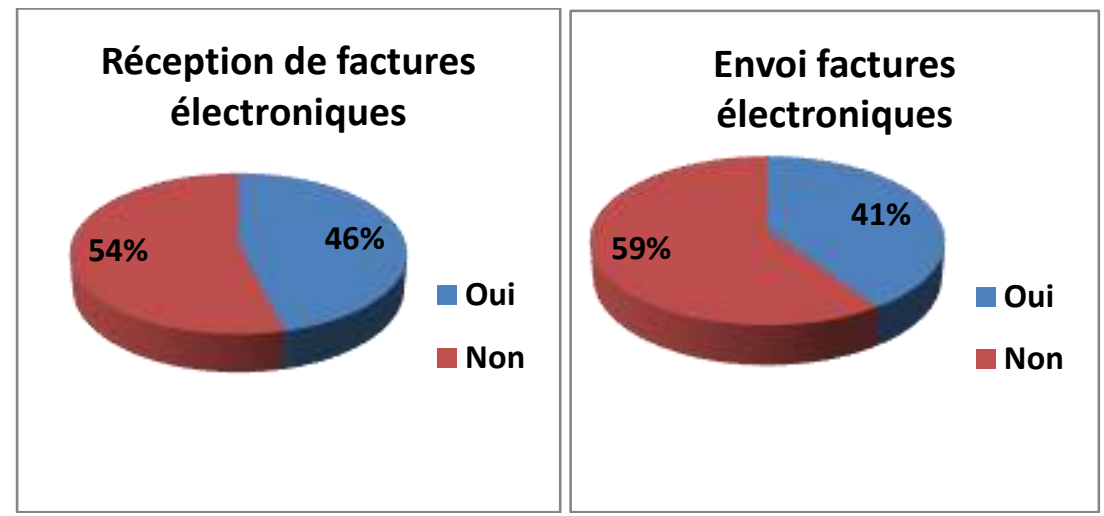

L'usage d'internet, plus précisément le courrier électronique représente aujourd'hui un moyen incontournable de communication entre les professionnels.

S'agissant de l'envoi et la réception des factures électroniques, les résultats de l'enquête permettent de constater qu'environ 46\% des PME font recours à l'envoi des factures par internet et $41 \%$ d'entre elles réceptionnent des factures électroniques.

Ces résultats, permettent de constater une prise de conscience des PME quant à la nécessité d'utiliser la facture électronique. Mais il reste néanmoins, une part importante des PME qui continuent à utiliser la facture traditionnelle (envoi postal ou portée). Ceci peut être expliqué par la disparité des moyens logistiques des PME, notamment le niveau d'informatisation et de connexion à internet.

b. L'ordre d'achat électronique :

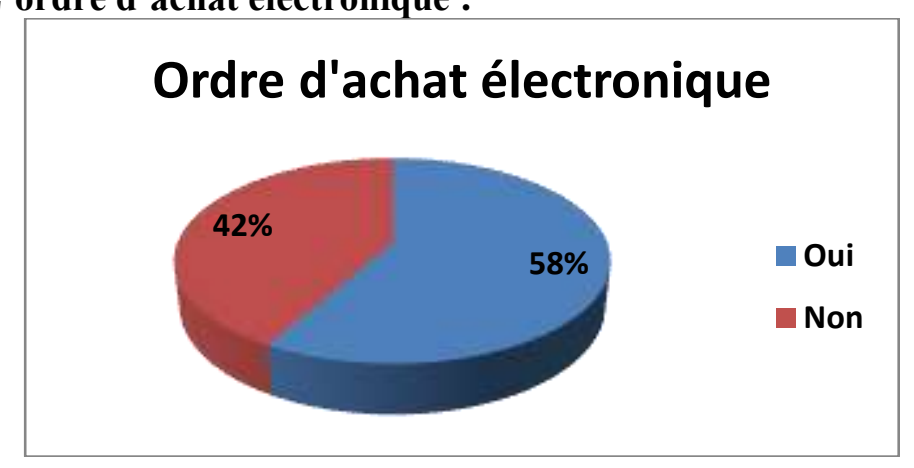

Le passage de l'ordre d'achat électronique peut être appréhendé comme une alternative qui répond aux besoins de flexibilité d'approvisionnements des entreprises.

La majorité des entreprises de grande taille interviewées ont recours au passage d'ordre d'achat par internet, soit 58\% de l'échantillon. Les PME semblent intégrer progressivement le facteur temps dans leur gestion commerciale (travailler en temps réel). 


\section{c. La gestion des contrats :}

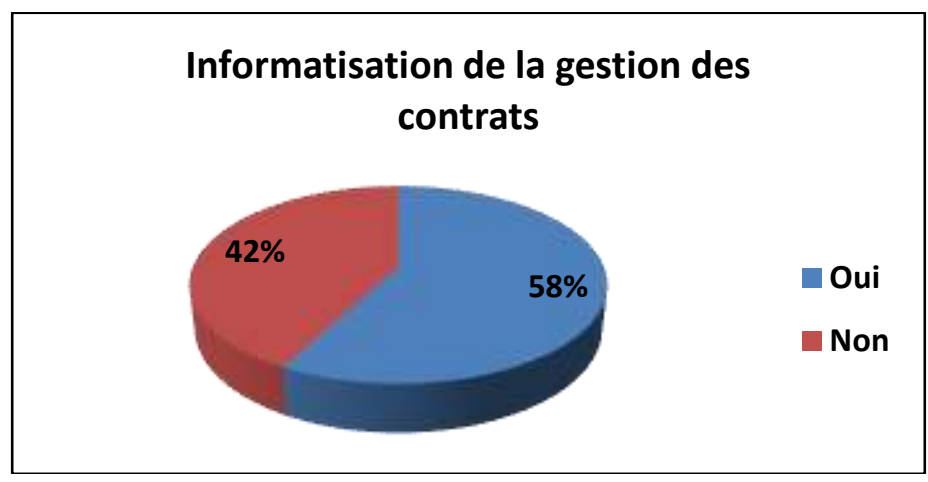

Les résultats de l'enquête permettent de voir que 58\% des PME ont informatisé la gestion de leurs contrats. C'est une prise de conscience des PME quant à la maitrise et le suivi de leurs contrats.

d. La logistique interne :

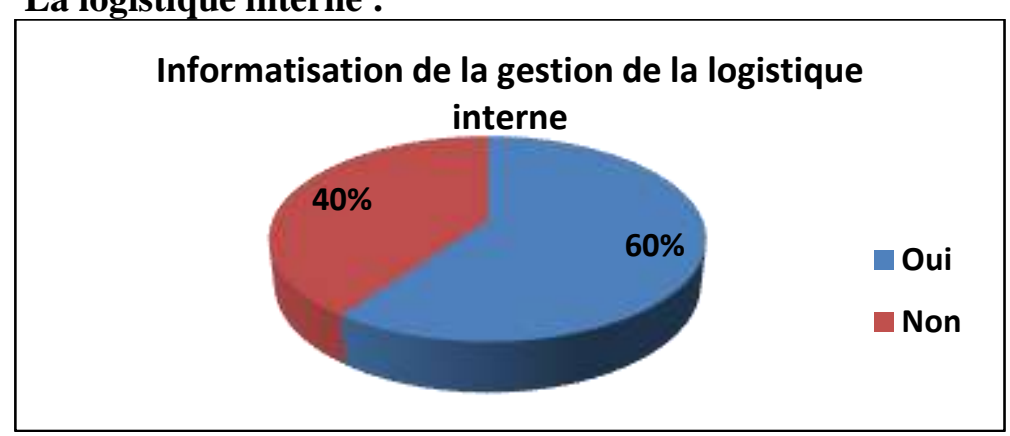

Plus de la moitié des PME de l'échantillon ont utilisé les TIC dans leur logistique interne soit $60 \%$ des entreprises. Ces résultats permettent de voir que les entreprises, semblent avoir compris les limites de la gestion traditionnelle, basée sur les supports manuels, très peu intégrée, de leurs moyens logistiques.

\section{e. Le processus de distribution :}




\section{Informatisation du processus de distribution}

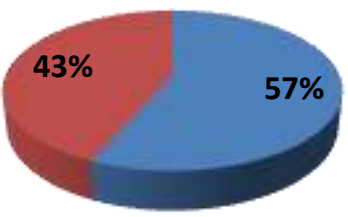

L'informatisation du processus de distribution qui va de la sortie du produit fini jusqu'à l'arrivée de ce dernier aux intermédiaires ou au client final permet d'avoir une meilleure vision et surtout, une traçabilité optimale de la distribution. Les résultats de l'enquête permettent de voir que 57\% des PME ont eu recours à l'informatisation du processus de distribution, indispensable à une gestion efficace du réseau et qui signifie satisfaction des clients et amélioration de la position commerciale et de part de marché.

\section{f. Le fichier clientèle :}

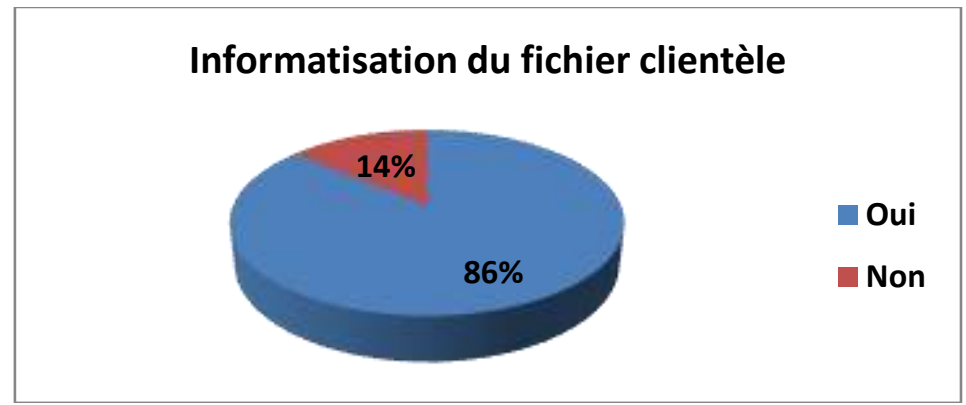

L'informatisation du fichier clientèle représente un moyen qui permet aux entreprises d'être à l'écoute de leur clientèle et être donc en mesure de proposer à cette dernière des produits adaptés à leurs besoins. $86 \%$ des entreprises touchées par l'enquête déclarent disposer d'un fichier informatisé de leur clientèle. L'analyse de ces données explique aussi, le souci de la PME algérienne de mettre en place des canaux qui lui permettent d'être constamment à l'écoute de son marché.

g. Indice de pénétration des TIC dans la chaine logistique :

Pour les besoins de l'enquête, nous avons calculé un indice global de pénétration des TIC de notre échantillon de PME.

Pour le calcul de cet indice nous avons appliqué en fonction de l'importance des variables, la pondération suivante :

- Niveau 1 (x 1) pour : les entreprises qui n'ont introduit les TIC dans la chaine logistique ; 
- Niveau 2 (x 2) pour: les entreprises qui ont recours à la facture électronique et la gestion des contrats informatisées;

- Niveau 3 (x 4) pour : les entreprises, qui en plus de l'utilisation des factures électroniques et une gestion informatisée des contrats, ont introduit les TIC dans leur logistique interne;

- Niveau 4 (x 6) pour: les entreprises qui ont recours à la facture électronique, à une gestion informatisée des contrats, à une logistique interne informatisée et à un fichier clientèle informatisé ;

- Niveau 5 (x10) pour : les entreprises qui utilisent les TIC dans les factures, la gestion des contrats, la logistique interne, la gestion du fichier clientèle et la logistique externe.

L'indice de pénétration moyen des TIC dans la chaine logistique est alors calculé en additionnant les scores et en les divisant par le total des coefficients de pondération.

\begin{tabular}{|c|c|c|c|c|}
\hline Niveaux & $\begin{array}{c}\text { Nombre } \\
\text { d'entreprise }\end{array}$ & Coefficient & $\begin{array}{c}\text { Note de } \\
\text { pondération }\end{array}$ & $\begin{array}{c}\text { Indice de } \\
\text { pénétration }\end{array}$ \\
\hline Niveau 1 & 185 & 1 & 185 & $8 \%$ \\
\hline Niveau 2 & 42 & 2 & 84 & $4 \%$ \\
\hline Niveau 3 & 30 & 4 & 120 & $5 \%$ \\
\hline Niveau 4 & 26 & 6 & 156 & $7 \%$ \\
\hline Niveau 5 & 21 & 10 & 210 & $9 \%$ \\
\hline Total & $I$ & $\mathbf{2 3}$ & $\mathbf{7 5 5}$ & $\mathbf{3 3 \%}$ \\
\hline
\end{tabular}

Nous avons obtenu un indice de pénétration moyen des technologies de l'information et de la communication de l'ordre de 33\%.

L'analyse des indices de pénétration des technologies par niveau, permet de voir que le taux des entreprises qui ont introduit les TIC dans tous les domaines est le plus important $(9 \%)$, viennent ensuite les entreprises qui n'ont pas informatisé leur logistique $(8 \%)$.

III. Impact des TIC sur la croissance et la position concurrentielle. 
1. La croissance :

a. Chiffre d'affaires.

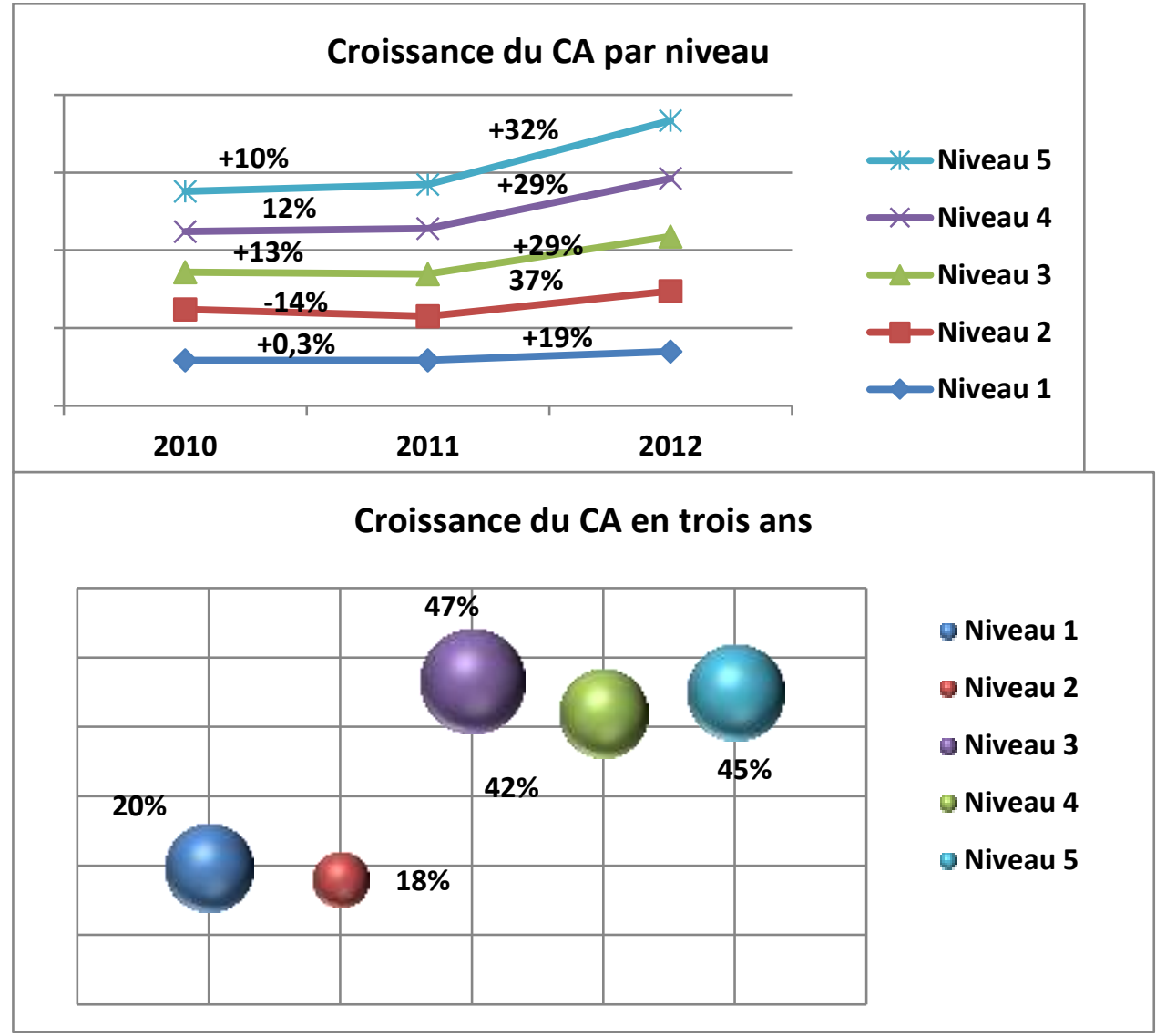

L'analyse de la croissance du chiffre d'affaires par niveau, permet de voir que les entreprises de niveau 3, 4 et 5 enregistrent un taux de croissance en trois ans le plus élevé, soit respectivement de : $47 \%, 42 \%$ et $45 \%$.

Ceci permet de dire que plus les entreprises investissent et utilisent les technologies de l'information et de la communication dans la chaine logistique et plus elles réalisent une augmentation dans le chiffre d'affaires.

b. Valeur ajoutée. 
Entreprises manufacturières ; TIC et chaine logistique L'impact des TIC sur l'avantage concurrentiel de la chaine logistique dans les entreprises manufacturières Algériennes

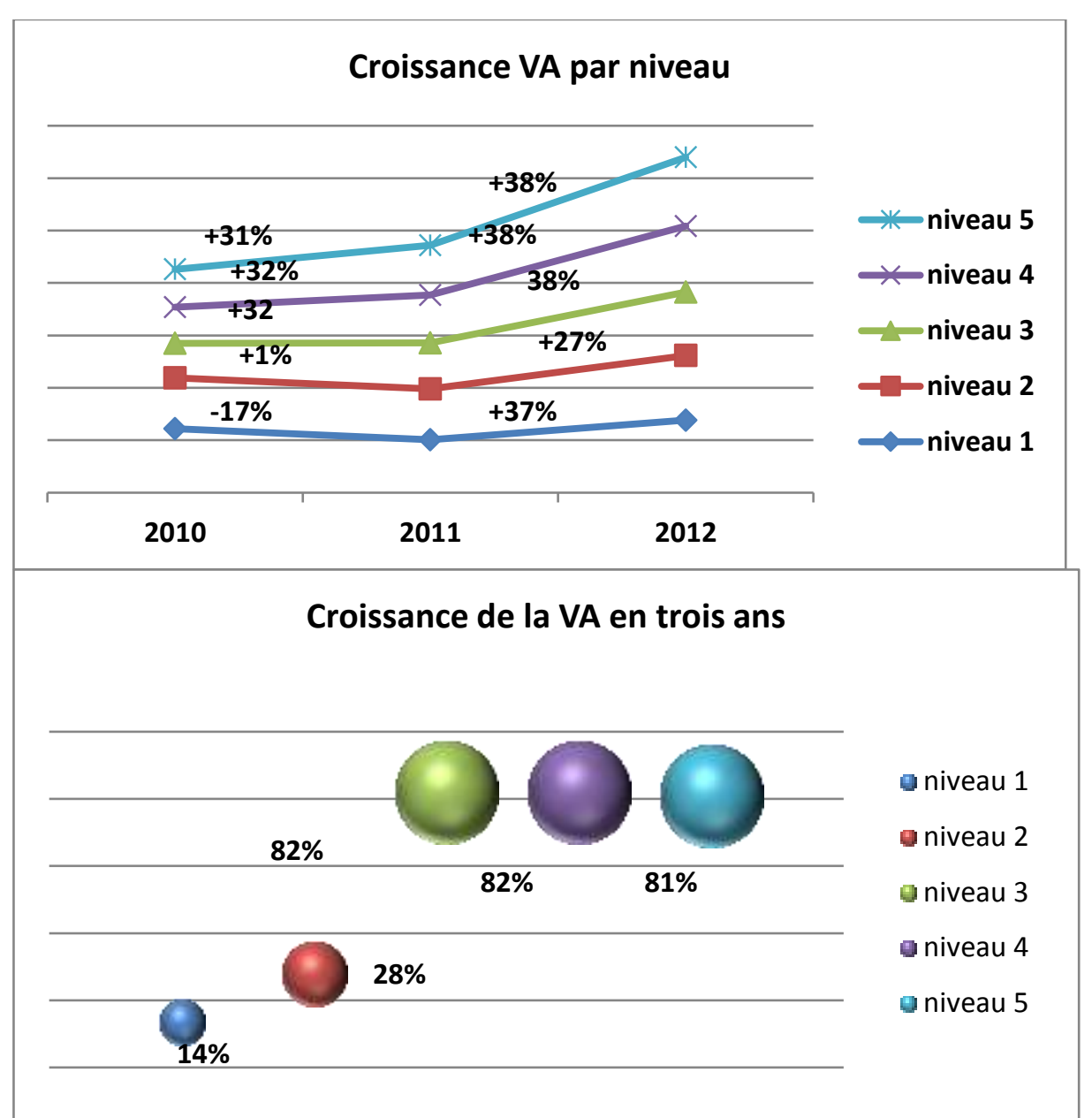

En ce qui concerne, la variation de la valeur ajoutée, appréhendée par niveau sur trois ans, l'analyse de cette dernière permet de voir que les entreprises de niveau 1 enregistrent une croissance assez faible (14\%) par rapport aux entreprises de niveau $3(82 \%), 4(82 \%)$ et $5(81 \%)$.

Ces résultats mettent en évidence la relation étroite qui existe entre l'accroissement de la valeur ajoutée par l'entreprise et l'élargissement des domaines d'introduction des TIC dans la chaine logistique. L'élargissement des domaines des TIC au sein de l'entreprise, est suivi automatiquement d'une élévation de la valeur ajoutée par cette dernière. 
c. Appréciation des ventes.

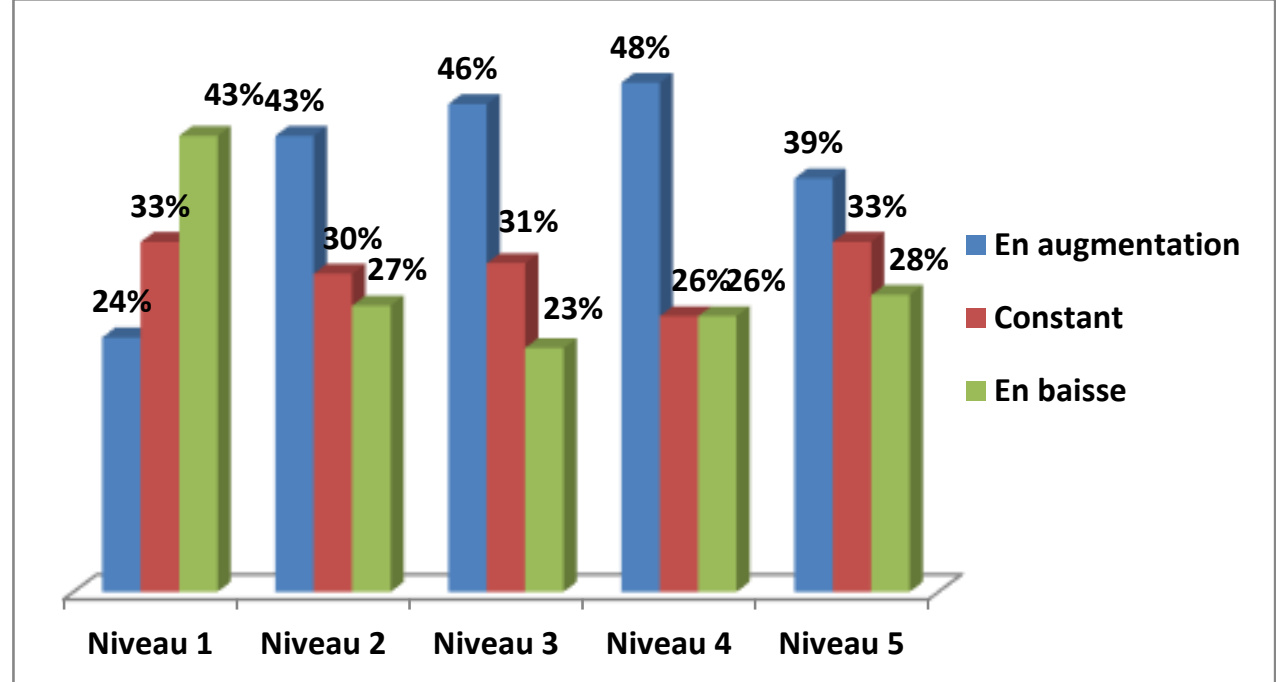

L'appréciation des ventes, met en avant la corrélation entre l'informatisation de la chaine logistique des entreprises et la variation positive de leur volume des ventes.

En effet, la part des entreprises qui ont vu leurs ventes augmentées, est importante dans celles du niveau $3(43 \%), 4(46 \%)$ et 5 (48\%). On constate en parallèle, que la part des entreprises, dont les ventes ont diminué, est devenue faible dans ces entreprises.

\section{Position concurrentielle.}


a. Positionnement.

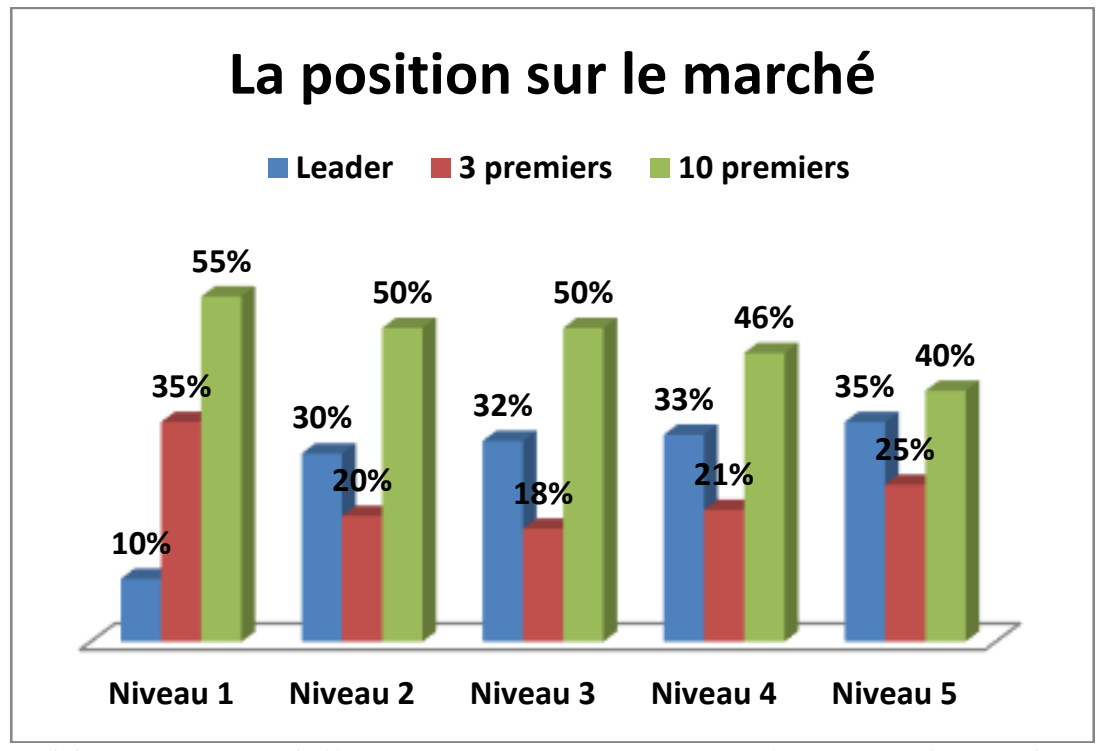

Pour la position concurrentielle occupée, on constate que les entreprises qui ont introduit les TIC dans leur chaine logistique occupent majoritairement les dix premières places sur le marché. On observe d'autre part, que les parts de marché des entreprises leaders (voire les trois premières sur leur marché) augmentent parallèlement à l'élargissement des domaines de fonctions touchées par les TIC soit les parts des entreprises de niveau 3,4 et 5 qui occupent les places de leaders sont respectivement de : $32 \%, 33 \%$ et $35 \%$.

\section{b. Proximité.}




\section{Proximité : Fournisseurs}

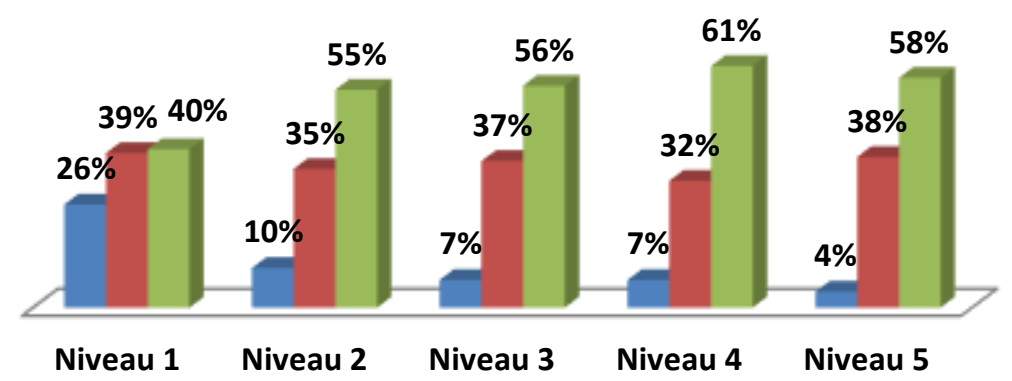

Même wilaya $\quad$ National Etranger

Notre échantillon d'enquête est basé sur un circuit d'approvisionnement assez large, qui ne s'arrête pas à une échelle locale (wilaya), bien au contraire, le champ d'étude est élargi aux sources d'approvisionnement, nationales et internationales.

Une part importante des entreprises de niveau 2,3 4 et 5, s'approvisionnent auprès de fournisseurs étrangers avec des taux respectives de : 55\%, 56\%, 61\% et $58 \%$.

Les technologies de l'information et de la communication (Porter \& Millar, 1985) utilisées dans la chaine logistique permettent aux opérateurs qui se sont dotés de ces outils, d'élargir le champ de recherche de fournisseurs pour s'approvisionner aux meilleures conditions de prix, de qualité de la matière et de transport. L'intégration des TIC dans le système d'approvisionnement permet aussi à l'entreprise de travailler avec des fournisseurs géographiquement éloignés, d'échanger des informations en temps réel et de faciliter la communication entre eux. Ce qui constitue pour ces entreprises, un atout à même de contribuer à une réduction des coûts de production. 


\section{Proximité avec les clients}

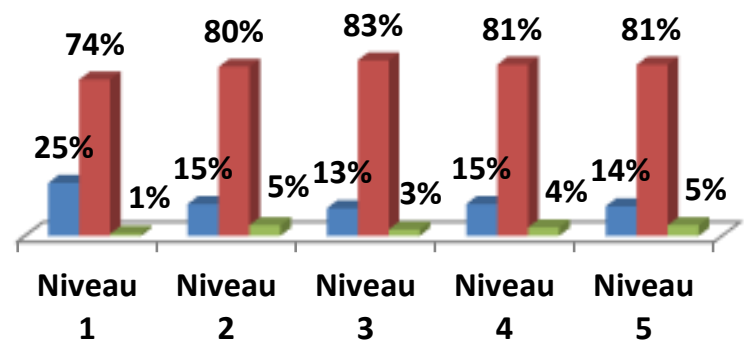

Même wilaya

Niveau National

A l'étranger

Les résultats de l'enquête montrent que les PME de notre échantillon d'enquête, s'adressent aussi bien à une clientèle résidente qu'à une clientèle située à l'étranger.

On constate que l'élargissement géographique de la clientèle (national et étranger) est lié au degré d'intégration des TIC dans la chaine logistique de ces entreprises. Plus l'intégration des TIC dans la chaine logistique est importante chez les entreprises et plus la part de clientèle de ces entreprises est importante. En effet, on enregistre un taux qui varie entre $81 \%$ et $83 \%$ pour les entreprises qui ont une clientèle locale et qui ont eu recours à l'informatisation de la chaine logistique. Ces taux sont un peu en augmentation par rapport à celui des entreprises qui n'ont pas intégré les TIC de la chaine logistique.

Pour les entreprises qui s'adressent à une clientèle étrangères, on constate que le taux est faible pour les entreprises qui n'ont pas informatisé leur logistique par rapport à celle qui l'on informatisé.

Ainsi, est-il permis d'affirmer que les technologies de l'information et de la communication rendent le marketing plus attractif pour les clients et permettent ainsi aux entreprises d'élargir leur circuit de distribution aux niveaux national et international, d'entreprendre des actions durables d'information et de conseil en direction des consommateurs à des coûts relativement faible.

\section{c. Nature des relations.}




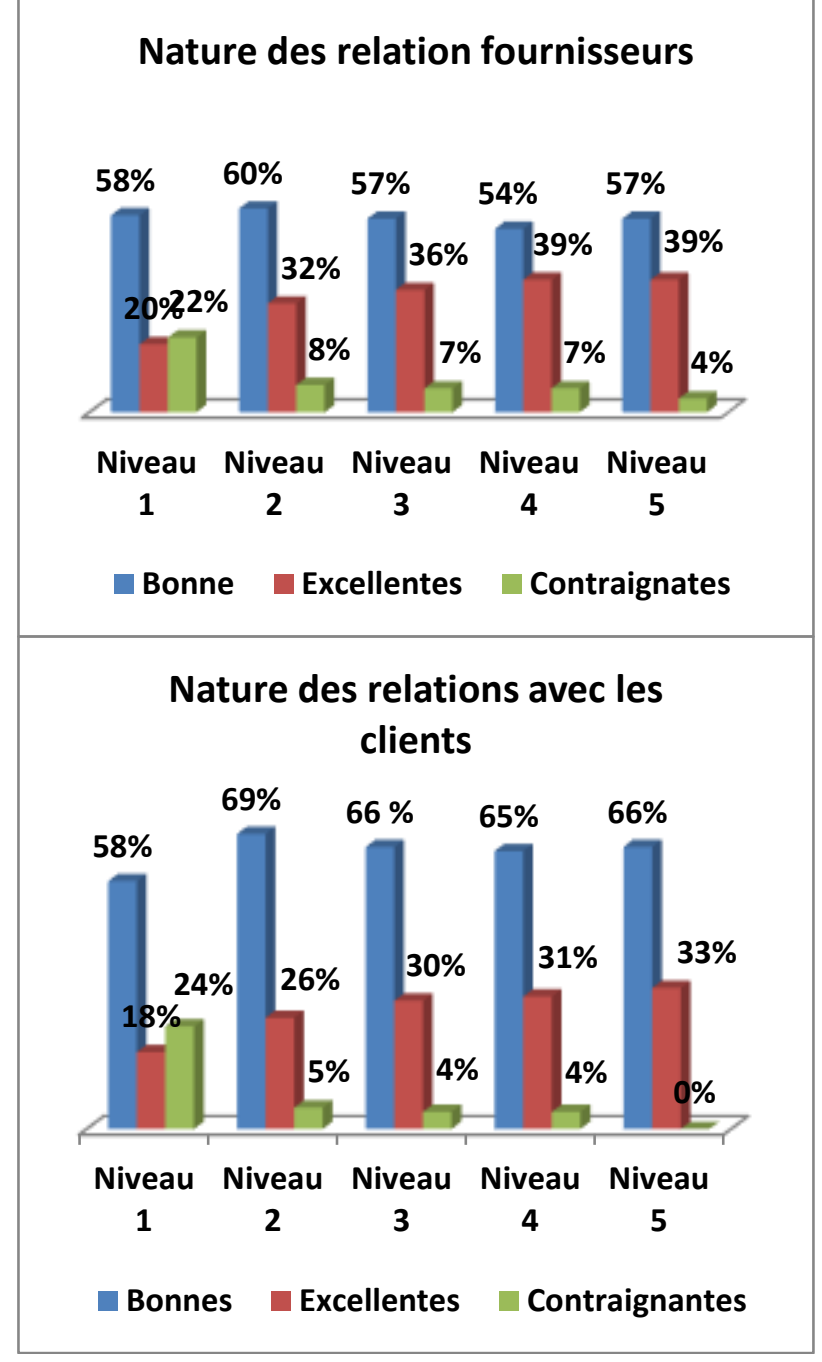

Une analyse de la nature des relations qu'entretient l'entreprise avec ses partenaires, permet de constater que les entreprises issues de notre échantillon de PME, développent de bonnes relations avec leurs fournisseurs et leurs clients.

On constate que la part des entreprises qui ont des relations contraignantes avec leurs fournisseurs et leurs clients, est beaucoup plus faible dans les entreprises de niveau 3, 4 et 5 .

On relève aussi que les entreprises qui ont intégré les TIC dans divers domaines de la chaine logistique (niveau 3, 4 et 5), enregistrent de bonnes relations voire d'excellents avec leur fournisseurs et leurs clients. 
Aussi, est-il permis de noter que les TIC assurent un travail collaboratif avec les maillons de la chaine logistique à savoir les fournisseurs et les clients ; Ce qui permet de développer entre partenaires, des relations commerciales innovantes et profitables.

\section{Conclusion :}

Dans cette recherche, nous avons tenté de voir d'une part l'importance accordée par les entreprises algériennes à l'introduction des TIC dans leur chaine logistique et d'autre part le rôle joué par l'introduction des TIC au sein de la chaine logistique, sur la compétitivité des entreprises algériennes.

L'analyse de l'impact de l'usage des TIC dans la chaine logistique sur la création de valeur pour le client, nous a permis de voir que sur l'échantillon des entreprises algériennes de secteur manufacturé (324 entreprises), la part de celles qui disposent des TIC est importante (256 entreprises): soit 29 grandes entreprises et 227 PME.

En ce qui concerne, l'usage des TIC dans la chaine logistique dans les PME, nous avons pu constater que divers domaine de la logistique amont et aval sont concernés par cette introduction (facture électronique, ordre d'achat électronique, gestion des contrats, logistique interne, processus de distribution, gestion du fichier clientèle).

L'analyse de la compétitivité réalisée par les entreprises qui utilisent les TIC dans leur chaine logistique, a permis de voir que les PME, qui ont informatisé plus de trois domaines logistique (niveau 3 et 4), ont vu leur croissance s'amélioré en enregistrant une variation des ventes plutôt en augmentation ou constante. Ces dernières enregistrent aussi une bonne position concurrentielle. Une position traduite trois principaux points : premièrement par l'occupation des places de leader voir de trois premiers, deuxièmement par une importante proximité entre les entreprises et leurs partenaires et troisièmement par de relations bonnes entre les entreprises et leurs partenaires.

Cette recherche permet de voir que les entreprises algériennes qui ont introduits les TIC dans leur chaine logistique enregistrent une meilleure performance/ compétitivité. Mais il serait plus intéressant d'analyser d'autres aspects qui permettent de consolider encore plus ces résultats. L'étude de l'aspect sociétal représente une dimension de plus en plus indispensable à étudier. En effet, après avoir attendu des technologies info-logistiques qu'elles procurent des gains de productivité, on attend désormais de leur mise en œuvre qu'elles réduisent l'empreinte écologique des activités de logistique et de transport.

\section{Bibliographie :}


(1)- Arcis, L. (1999). L'impact des ERP sur la chaîne logistique. Logistique § Management, pp. 26-42.

(2)- Beamon, B. (1998). Supply chain design and analysis : models and methods. International journal of production economics, pp. 28-94.

(3)- Berraki Hanifa (2014) : " étude de la chaine logistique de l'entreprise Danone Djurdjura Algérie'. Mémoire en vue de l'obtention du diplôme en science, Institut Agronomique Méditerranéen de Montpellier IAM.

(4)- Boudahri Fethi (2013) : 'Conception et pilotage d'une chaine logistique agroalimentaire : application produits de volaille dans la ville de Tlemcen'. Thèse en vue de l'obtention du diplôme de doctorat en sciences spécialité : productique, Université Abou-Bekr Belkaid-Tlemcen.

(5)- Delfmann, W., \& Gehring, M. (2003). Le rôle des technologies de l'information dans la performance logistique. Logistique $\S$ Management, pp. 510.

(6)- Fabbe-Costes, N. (2002). Evaluer la création de valeur du supply chain management. Logistique § Management, pp. 29-36.

(7)- Fabbes-Costes, N., \& Meschi, P. (2000). Situations types et évolutions de la logistique dans l'organisation. Logistique § Management, pp. 101-112.

(8)- Fernandes, V. (2007). Gestion de la relation fournisseurs : étude des relations entre les modes de contrôle et les dimensions des transactions . ComptabilitéContrôle-Audit.

(9)- Giunipero, L., \& Brand, P. (1996). Purchasing's role in supply chain management. The international journal of logistics management, pp. 29-38.

(10)-Harrington, L. (1999). Supply chain management: Overview of supply chain operations references model. Industry week .

(11)-Jobin, M., \& Friel, T. (2001). Sur la piste de la création durable de valeur à travers la chaîne logistique. Logistique § Management, pp. 27-33.

(12)- Laurentie, J., Berthelemy, F., Gregoire, L., \& Terrier, C. (2000). Supply chain management. Processus et méthodes logistiques . AFNOR.

(13)- Lorino, P. (1997). Méthodes et pratiques de la performance: Le guide du pilotage. Paris: Les éditions d'organisation.

(14)- Mehrabikoushki Ali (2008) : Partage d'information dans la chaîne logistique : 'Evaluation des impacts sur la performance d'une chaîne logistique des modes de collaboration mis en œuvre entre les partenaires et des informations échangées " en vue de l'obtention du diplôme de doctorat en Génie informatique, L'Institut National des Sciences Appliquées de Lyon.

(15)- Morvan, A. (2005). Mise en place d'un ERP sur une supply chain intégrée : l'exemple de HILTI. Logistique § Management, pp. 21-29.

(16)- Nakhla, M. (2006). Supply chain management et performance de l'entreprise : Value Based Supply Chain. Logistique § Management, pp. 65-77. 
(17)- Palevich, R. (1997). Supply chain management: International conference proceedings of APICS. The educational society for resource management, $\mathrm{pp}$. $1-5$.

(18)- Parsons, G. (1983). Information technology : a new weapon. Sloan management review, pp. 3-13.

(19)- Porter, M. (1985). Competitive advantage: Creating and sustaining superior performance. New york: The free press.

(20)- Porter, M., \& Millar, V. (1985, Juillet / Août). How information gives you competitive advantage. Harvard business review , pp. 149-160.

(21)- Primo, M., \& Amundson, S. (2002). An explotatory study of the effects of supplier relationships on new product development outcomes. Journal of operations management, pp. 33-52.

(22)- Ripoll, G. (1997). L'ECR : Jusqu'ou peut-on aller dans l'intégration de la chaîne logistique? Logistique § Management, pp. 15-21.

(23)- z'eroual, T., Blanquart, C., \& Carbone, V. (2011). Supply chain management : Portee et limites. Les cahiers de recherches de l'ESCE.

\section{Notes :}

(1)- Porter, M., \& Millar, V. (1985). How information gives you competitive advantage. Harvard business review , pp. 149-160.

(2)- Porter, M. (1985). Competitive advantage: Creating and sustaining superior performance. The free press .

(3)- Ripoll Gabriel, «L'ECR : Jusqu'où peut-on aller dans l'intégration de la chaîne logistique ? », Logistique\& Management, Vol. 5 - $\mathrm{N}^{\circ} 1$, 1997, p 15-21.

(4)- DELFMANN Werner, Martin GEHRING, " Le rôle des technologies de l'information dans la performance logistique', Logistique \& Management, Vol. $11-\mathrm{N}^{\circ} 1,2003$

(5)- MORVAN Arnaud, ' Mise en place d'un ERP sur une supply chain intégrée : l'exemple de HILTI', Logistique \& Management, Vol. 13 - $\mathrm{N}^{\circ} 2,2005$

(6)- Boudahri Fethi (2013) : 'Conception et pilotage d'une chaine logistique agroalimentaire : application produits de volaille dans la ville de Tlemcen'. Thèse en vue de l'obtention du diplôme de doctorat en sciences spécialité : productique, Université Abou-Bekr Belkaid-Tlemcen.

(7)- Berraki Hanifa (2014): " étude de la chaine logistique de l'entreprise Danone Djurdjura Algérie'. Mémoire en vue de l'obtention du diplôme en science, Institut Agronomique Méditerranéen de Montpellier IAM

(8)- Oliver, R.K. et Webber, M.D. (1982), Supply Chain Management: Logistics catches up with strategy, dans Christopher, M.G., Logistics, The strategic issue, London, Chapman and Hall

(9)- BEAMON B.M. Supply chain design and analysis: models and methods. International Journal of Production Economics, 1998, vol. 55, pp. 281-94. 
(10)- Lorino P. (1997), Méthodes et pratiques de la performance. Le guide du pilotage, Les éditions d'organisation, Paris.

(11)- Fabbe-Costes N., (2002), Évaluer la création de valeur du supply chain management, Logistique \& Management, Vol.10, $\mathrm{n}^{\circ} 1$.

(12)- Porter M.E. (1985) Competitive Advantage. Creating and Sustaining Superior Performance, The Free Press, New York

(13)- GIUNIPERO L.C., BRAND R.R. Purchasing's role in supply chain management. The International Journal of Logistics Management, 1996.

(14)- HARRINGTON, L.H. Supply chain management: overview of supply chain operations references model. Industry Week, 1999, vol. 248, n 2.

(15)- PALEVICH R.F. Supply chain management. International Conference Proceedings of APICS 1997-The educational Society for Resource Management, 1997, pp. 1-5.

(16)- Alber et Walker 1997, in Ali Mehrabikoushki, «Evaluation des impacts sur la performance d'une chaîne logistique des modes de collaboration mis en oeuvre entre les partenaires et des informations échangées », 2008.

(17)- LAURENTIE J., BERTHELEMY F., GREGOIRE L., TERRIER C., Processus et méthodes logistiques - Supply chain management, AFNOR, 2000 (18)- Fabbes-Costes N., Meschi P. X., situations types et évolutions de la logistique dans l'organisation, logistique et management, vol. 8, $\mathrm{n}^{\circ} 1,2000$, pp101-112.

(19)- JOBIN Marie-Héléne, FRIEL Terri, ' Sur la piste de la création durable de valeur à travers la chaîne logistique", Logistique et Management, Vol. 9 $\mathrm{N}^{\circ} 2,2001$;

(20)- Primo M. A. M., Amundson S. D., "An exploratory study of the effects of supplier relationships on new product development outcomes", Journal of Operations Management, vol. 20, $\mathrm{n}^{\circ}$ 1, 2002, p. 33-52.

(21)- NAKHLA Michel," Supply Chain Management et performance de l'entreprise "Value Based Supply Chain Management Model", Vol. $14-\mathrm{N}^{\circ} 1$, 2006.

(22)- Boudahri Fethi (2013) : 'Conception et pilotage d'une chaine logistique agroalimentaire : application produits de volaille dans la ville de Tlemcen'. Thèse en vue de l'obtention du diplôme de doctorat en sciences spécialité : productique, Université Abou-Bekr Belkaid-Tlemcen.

(23)- Berraki Hanifa (2014) : " étude de la chaine logistique de l'entreprise Danone Djurdjura Algérie". Mémoire en vue de l'obtention du diplôme en science, Institut Agronomique Méditerranéen de Montpellier IAM.

(24)- Fernandes Valérie, "Gestion de la relation fournisseurs : étude des relations entre les modes de contrôle et les dimensions des transactions », Comptabilité - Contrôle - Audit, 2007/2 Tome 13, p. 53-74. 
(25)- Thomas Z'eroual, Corinne Blanquart, Valentina Carbone. SUPPLY CHAIN MANAGEMENT : PORTEE ET LIMITES - L'apport des th'eories des r'eseaux -. Les cahiers de recherche de l'ESCE, 2011, pp.20

(26)- Parsons G.L. (1983), "Information technology: a new weapon", Sloan Management Review, 25, 1, 3-13.

(27)- Ives B. ET Learmonth G. (1984), "The information system as a competitive weapon", Communications of the ACM, 27, 12, 1193-1201.

(28)- Porter M.E. et Millar V. (1985), "How information gives you competitive advantage", Harvard Business Review, juillet/août, 149-160.

(29)- Ripoll Gabriel, «L'ECR : Jusqu'où peut-on aller dans l'intégration de la chaîne logistique ? », Logistique\& Management, Vol. 5 - $\mathrm{N}^{\circ} 1$ 1, 1997, p 15-21.

(30)- ARCIS Laurent, « L'impact des ERP sur la chaîne logistique », Logistique \& Management, Vol. $7-\mathrm{N}^{\circ} 1,1999, \mathrm{p} 26-42$.

(31)- DELFMANN Werner, Martin GEHRING, " Le rôle des technologies de l'information dans la performance logistique", Logistique \& Management, Vol. $11-\mathrm{N}^{\circ} 1,2003$

(32)- MORVAN Arnaud, "' Mise en place d'un ERP sur une supply chain intégrée : l'exemple de HILTI', Logistique \& Management, Vol. 13 - ํ2, 2005 